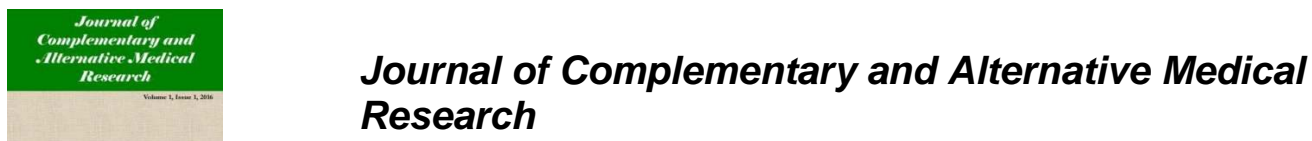

3(1): 1-14, 2017; Article no.JOCAMR.32941

S

\title{
Tui Na (or Tuina) Massage: A Minireview of Pertinent Literature, 1970-2017
}

\author{
Abdullah Mohammed Al-Bedah ${ }^{1}$, Gazzaffi Ibrahim Ali ${ }^{1}$ \\ Tamer Shaban Abushanab ${ }^{1}$ and Naseem Akhtar Qureshi ${ }^{1 *}$
}

${ }^{1}$ National Center for Complementary and Alternative Medicine, Ministry of Health, Riyadh, Saudi Arabia.

Authors' contributions

This work was carried out in collaboration between all authors. All authors equally contributed to the concept and design and drafted this paper. All authors revised this paper and furthermore author $N A Q$ put inputs in the final draft. All authors read and approved this paper for publication.

Article Information

DOI: $10.9734 / J O C A M R / 2017 / 32941$

Editor(s):

(1) Francisco Cruz-Sosa, Metropolitan Autonomous University Iztapalapa Campus Av. San Rafael Atlixco, Mexico.

Reviewers:

(1) Timothy Hui, Loma Linda University, California, USA (2) Parth Trivedi, C.M. Patel College of Physiotherapy, Gujarat, India. (3) Mario Bernardo-Filho, Universidade do Estado do Rio de Janeiro, Brazil. (4) Ana Carolina Urbaczek, University of São Paulo, Brazil. (5) Yu-Ching Hsu, Tainan Hospital, Ministry of Health and Welfare, Tainan, Taiwan. Complete Peer review History: http://www.sciencedomain.org/review-history/19376

Mini-review Article

Received 24 $4^{\text {th }}$ March 2017 Accepted $31^{\text {st }}$ May 2017 Published $6^{\text {th }}$ June 2017

\section{ABSTRACT}

Background: Tuina massage is a traditional method used effectively in the treatment of various ailments in China since ancient time, and currently it is used around the world.

Objective: This minireview aims to describe several aspects of Tuina massage an integral part of Traditional Chinese Medicine in order to fill up the knowledge gap concerning traditional practitioners in Saudi Arabia.

Methods: Electronic searches of databases using Boolean operators and keywords were conducted to retrieve data published in English and Chinese literature. Thousands of articles were identified and screened by two independent reviewers using exclusion and inclusion criteria, and 56 articles were finally included in this study.

Results: Tuina has a very rich history in Chinese culture since antiquity. With continuous 
advancements in research, training, regulation and clinical practice, Tuina massage became popular worldwide and now used either alone or in conjunction with other complementary and alternative medicine or conventional therapies in diverse diseases associated with pain and other symptoms with good outcome. Although Tuina has good safety profile with level of evidence (LOE) of I to III, well defined indications and contraindications, a variety of minor adverse effects together with some major complications including deaths have been reported in the literature. Besides continuous training of Tuina practitioners, Tuina massage practice needs regulatory measures and guidelines for avoiding complications and improving the clinical outcome of patients.

Conclusion: Evidently, Chinese Tuina massage supported by theory, mechanisms, procedure and included randomized clinical trials snapshots, systematic reviews and meta-analysis with LOE of I to III is reported to be effective in several conditions. Further, rigorous randomized controlled studies with active comparators including other traditional modality or conventional medications or placebo with intensified quality control measures are required to provide further robust evidencebased data to support its efficacy in chronic diseases associated with pain and disabilities.

Keywords: Chinese Tuina massage; Traditional Chinese medicine; Tuina research; Tuina complications; Tuina indications and contraindications; training and regulation.

\section{INTRODUCTION}

Traditional Chinese massage literally means "pushing (and) grasping", and called Tui $\mathrm{Na}$ or Tuina (TN) in China. Tui $\mathrm{Na}$ is pronounced as "Twee Nah". It is a form of non-invasive, external Chinese massage of soft tissue. Recently, its modified version called innovative TN manipulation is introduced in Traditional Chinese Medicine (TCM). These Chinese massage remedies are the most popular TCM therapies used in a variety of diseases including joint pain, muscle weakness, facial paralysis, stomach problems and Parkinson's disease (PD) pain $[1,2]$. The earliest descriptions of Chinese massage were found in the Yellow Emperor's Inner Classic of Medicine, dating back more than 5000 years ago [1]. However, Tui $\mathrm{Na}$ did not occur in the Chinese (published) literature until the Ming dynasty (1368-1644 AD) where it first mentioned in a book on pediatric Tui $\mathrm{Na}$. However, it does not mean that Tuina was not used in adult as well as paediatric patients prior to Ming dynasty. Prior to this period, therapeutic massage was called "An Mo", which literally signifies "pressing (and) rubbing" (Tuina) and was practiced to relieve pediatric as well as adult suffering. Later in the Ming dynasty (1368-1644 AD), pediatric TN became an independent field of study. Notably, the first Tuina School was established in Shanghai, China. In Chinese massage or called Tuina, a wide range of technical manipulations are conducted by a practitioner at specific body locations [3,4]. For this purpose, the practitioner uses his finger, hand, elbow, knee, or foot that are applied to muscle or soft tissue [4]. Tui $\mathrm{Na}$ is also referred to as Chinese therapeutic body work [3] and sequential Yin TN, i.e., Chinese energy massage [2]. With continuous advances in Tuina especially in China, TN use was considered in emergency medicine. In the Sui dynasty (581-618 AD), TN became an independent area of study and the title of "manual therapy practitioner" began to be used [3]. Later Tuina was included in the medical education system in China. The department of Tuina massage (literal meaning pinch and pull) was one of the four clinical, medical departments in the Tang dynasty (618-907AD). Currently, Tuina is taught in many academic traditional universities and used globally [5]. Notably, Tuina is distinctively different from other manual therapies such as chiropractice, and a therapeutic method of TCM [3].

Traditional practitioners are impressively unaware of Tuina massage in Saudi Arabia. Furthermore to our knowledge, there is no published literature on Tuina in Saudi Arabia and other Gulf countries. This minireview on Tuina massage will partially fill the knowledge gap of traditional practitioners in Arabian Gulf countries together with a push to training of concerned professionals, its regulation and promotion of practice and continuous research in Tuina massage.

\section{METHODS}

\subsection{Search}

Boolean operators were used to search specific data (from 1970 to 2017) on Tuina massage. Electronic searches of three databases and three open access publishing houses (Google Scholar, PubMed, OvidSP and Dovepress.com, 
Hindawi.com and Sciencdomain.org) using keywords such as Tuina Massage OR Chinese Tui Na Massage OR Chinese massage therapy AND adverse effects OR complications OR Theory OR mechanisms OR procedures OR Case reports OR observational studies OR RCTs OR meta-analysis were conducted for retrieving articles published in English and Chinese literature. A large number of articles $(n=6952)$ were retrieved. Two authors (NAQ and TSA) independently reviewed the available data for extracting pertinent articles. Consequently, unrelated articles $(n=2005)$, inaccessible papers (because of high price tag) $(\mathrm{n}=2402)$, articles cited in systematic reviews and meta-analysis $(n=335)$, no abstract available $(n=42)$, duplications $(n=2075)$, and books $(n=28)$ were excluded. The remaining were 65 , which were screened further for eligibility, and those articles which used Tuina as standalone therapy and in conjunction with other traditional Chinese medicine and conventional therapies were finally included $(n=51)$ [6] (Fig. 1). Other inclusion criteria were types of studies, participants of interest, and outcome.

\section{RESULTS}

\subsection{Definition}

Tui Na or Tuina (in Chinese: 推拿) is a form of complementary and alternative medicine (CAM). It is a Chinese massage, manipulative and mobilization therapy often used in conjunction with acupuncture, moxibustion, fire cupping, Chinese herbalism, T'ai chi, and qigong [4]. Its literal meaning is "pinch and pull". Tuina includes a wide range of TCM therapeutic massage and body work including manipulation and mobilization [3]. In general, Tui $\mathrm{Na}$ is not used for pleasure or relaxation; however it is a treatment technique to address specific patterns of disharmony in qi energy flow in the body [5]. Chinese massage therapy has many forms of methods, and defining principles primarily based on acupressure points and balance of qi energy in the body. Overall, TN is an effective massage therapy with widening applications in CAM including conventional medicine but evades universal definition.
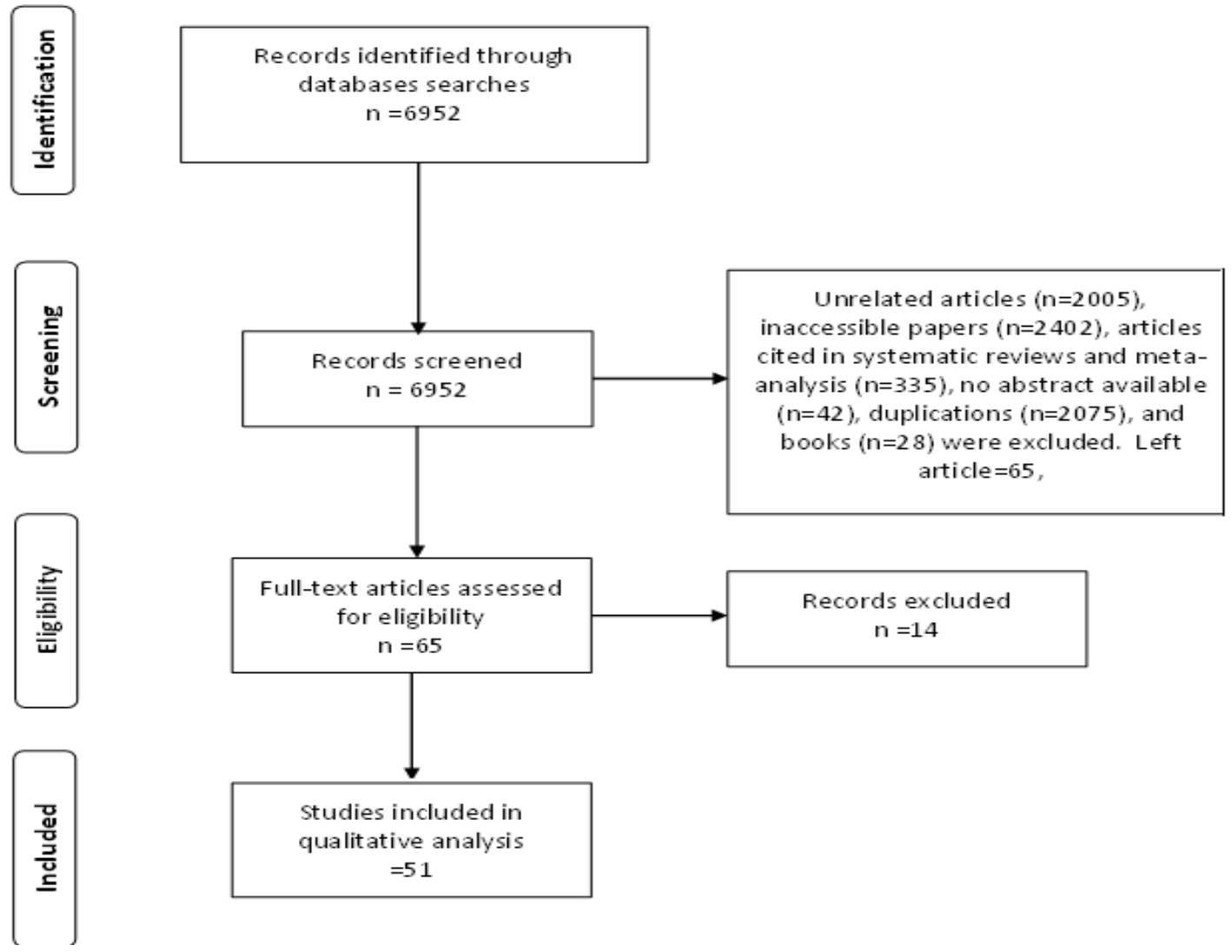

Fig. 1. Prisma flow diagram 


\subsection{Tui Na Classification}

According to traditional Chinese Medicine, Tui $\mathrm{Na}$ massage is categorized into: 1) Tuina massage: This type increases local blood circulation by removing stasis, and to improve the function of the related internal energy by regulating ill areas in the body, 2) Tuina manipulation: improved version of TN massage and this type includes manipulations such as kneading, rolling, pressing, plucking, and relaxing the affected/diseased areas and 3) Chinese massage (TN): this type is a combination of Tuina manipulation and mobilization (innovative Tui Na) [7]. Overall, in addition to this categorization, various terms have been used to describe TN [3-5,7] and possibly TN classification needs revision and further expansion and refinement.

\subsection{Tui Na Technique}

Every treatment modality has guiding technique for using it effectively. In Tuina, three massage techniques are used, which are: the first technique is to massage the soft tissue and stimulate acupoints in the meridian system; the second technique is the joint mobilization, which is commonly used in spine injury; the third technique is to guide the patients in practicing Qi Gong energy exercises such as Yang style Taiji Quan [1]. Tuina techniques include pushing (Tui) and grasping ( $\mathrm{Na}$ ) of soft tissue [5] and others are kneading, rolling, pressing, plucking, and relaxing [7]. In clinical practice, individual manipulations of Tuina are rarely used; rather, combined manipulations are applied. There are more than 110 diverse manipulations, though only 20-30 are commonly used [8]. Although some progress is made concerning standardization of Tuina technique (i.e., frequency, duration and force) [9], further advances are required to match the evolving newer techniques of Tuina massage around the world.

\subsection{Tui Na Theory}

The main theory of Chinese massage is the meridian system, which considers that there are many fixed channels of flowing qi energy interlaced as a network throughout the body $[10,11]$. Chinese massage uses hand and arm techniques to massage the soft tissue of the body, which stimulates acupoints in the meridian system and mobilizes the joints. Patients are also guided to practice qi Gong energy exercises such as Taiji Quan. The force used during Chinese massage is the main stimulating factor that activates the channels of the meridian system and modifies their functions. Chinese massage allows energy to flow harmoniously through these pathways, allowing the body to heal itself naturally $[10,12]$.

\subsection{Tui Na Procedure}

TN includes multiple manipulations applied at different affected places which are rolling manipulation, pressing manipulation, spinepinching manipulation and vibrating and oblique pulling manipulation [13]. Chinese TN massage uses hand and arm techniques to massage the soft tissue or muscles of the body. This procedure stimulates acupoints in the meridian system, precisely allows flow of stagnant energy, and mobilizes the joints. TN mostly helps body healing by natural way $[10,12,14]$ without using any concurrent conventional medications. Patient management includes various manual techniques, rehabilitative exercises, patient education and therapeutic advice. Furthermore, the community of practitioners of Tuina recognizes the importance of referring their patients to conventional health-care providers when necessary [5]. A typical session of Tuina massage may vary from thirty minutes to one hour. Overall, TN techniques and procedures are mostly based on the principles of TCM. The TCM principles that guide Tui $\mathrm{Na}$ practice include yin and yang, five elements, qi, blood and body fluids, and by the identification of syndromes and patterns $[5,11]$.

\subsection{Mechanism}

Tuina massage is based on the principle of $q i$ (vital energy) as described comprehensively in TCM. The vital energy is imbalanced among patients with diseases. Tui $\mathrm{Na}$ tends to develop a balance in qi and ultimately a smooth flow of energy in the body. According to TCM, for example, Yin and Yang are disturbed in primary dysmenorrhea, which may be balanced by TN and acupuncture [13] through manipulating different channels including DU (governing vessel) channel connected with other channels in the body. The various mechanisms underpinning Tuina therapy help improve coordination of $q i$ and blood, regulate the functions of the zang-fu organs, warm channel and expel cold, promote the circulation of qi and remove blood stasis, and to help eliminate the pathological changes and various functional disturbance caused by wind- 
cold, qi-stagnancy, and blood stasis [7]. Chinese massage is reported to have anti-inflammatory and analgesic effects, and could be potentially effective in conditions with pain due to inflammation in the elderly population with no or minor side effects [1,15]. In patients with Parkinson's disease who often report of injury to their feet, Tui $\mathrm{Na}$ massage is carried out at the foot on the side of the body most affected by PD with improvement in PD symptoms [2]. Injury to the feet is theoretically reported to cause PD [2]. Patients with chronic fatigue syndrome often have dysregulation of oxygen free radicals metabolism and Tuina tends to clean superfluous oxygen free radicals to alleviate fatigue. This process may be one of the mechanisms of Tuina in treating chronic fatigue syndrome [16]. In a nutshell, TN uses various manual techniques at the specified meridian points for mobilizing qi energy in the body, and also various supplementary therapies (exercises) for patients' healing through enhancing body resistance [5].

\subsection{Indications}

Tui $\mathrm{Na}$ is primarily used for neurological and musculoskeletal conditions. TN scope concerning medical diseases has largely expanded, and TN is now used in many conditions associated with pain, stress, and chronicity. The therapeutic effects of Tuina depend upon multiple factors which include TCM and biomedical diagnosis, selection of location, meridians and acupoints for the application of techniques, proper and effective application of the techniques. Notably, all these techniques are expected to regulate the functions of the meridians and the zang-fu organs. Some snapshots of important medical conditions that may be effectively managed by Tui $\mathrm{Na}$ massage alone or in conjunction with other TCM or conventional medicine are briefly described. We also included summary of some systematic reviews and meta-analyses concerning some specific physical conditions treated by Tuina therapy.

\subsubsection{Primary dysmenorrhea}

Thirty patients who received $\mathrm{TN}$ and acupuncture were compared with 30 control case who received only acupuncture, there were significant difference between the two groups $(p<0.05)$. Those who received combined treatment showed significant improvement in primary dysmenorrhea symptoms [13]. No adverse effects of Tuina combined with acupuncture were reported in this study.

\subsubsection{Carpal tunnel syndrome [CTS]}

CTS is a common condition and presents with intermittent numbness, tingling, and burning pain in the three radial digits of both hands due to compression on median nerve. Ninety eight cases of CTS were randomly divided into a treatment group received warm-needling plus Tuina relaxing manipulation were compared with a control group received as usual treatment (hormone block therapy and drug medication). There was a significant difference concerning cure rate between the treatment group (81.7\%) and control arm (47.4\%, $P<0.01)$. Acupuncture plus TN manipulation is an effective therapy for CTS [14]. No adverse effects were reported in this study.

\subsubsection{Cervical spondylosis (CS) of vertebral artery type}

Cervical spondylosis is a degenerative disease of cervical disc. 126 CS patients were randomly divided into test-treated with innovative Tuina manipulations and control groups treated with the routine Tuina manipulations. The outcome measures were clinical effects, treatment times, clinical symptoms, and cerebral blood flow. The response to the treatment was $100 \%$ in the test group and $88.71 \%$ in the control group. Patients in the test group required $(7 \pm 4)$ treatments before recovery, while those in the control group required $(15 \pm 7)$ treatments before recovery $(p<0.05)$. The clinical symptoms exhibited greater improvement in the test group compared to the control group $(p<0.05)$. There were no differences in cerebral blood flow between the two groups. Both innovative Tuina and routine Tuina manipulations produced satisfactory therapeutic results in vertebral artery type CS patients. However, the innovative manipulation was more effective in improving the functional symptoms, although there were no changes in the cerebral blood flow [7]. No adverse effects were reported in this study.

\subsubsection{Knee osteoarthritis (OA)}

Knee osteoarthritis is a common problem that presents with pain and restricted movements, and often affects elderly population. OA is multifactorial in nature. Thirty patients with knee $\mathrm{OA}$ received Chinese massage therapy (CMT) three times per week for 2 weeks were assessed using pre- and post-treatment Visual Analogue Scale (VAS) for pain, and pre- and post-treatment knee muscle strength by the 
BiodexMulti-Joint System 3. Isokinetic muscle strength measurements were performed at 60 degrees/sand 180 degrees/s. The peak torque (PT), peak torque/body weight (PT/BW), total work (TW), average power (AP), hamstring/quadriceps $(\mathrm{H} / \mathrm{Q})$, and range of motion (ROM) values were recorded separately for flexors and extensors (muscles of knee joint). CMT significantly improved knee pain as assessed by the VAS in patients with knee OA $(p<0.05)$. Post-treatment values were significantly greater than pre-treatment values in the extensor muscles for PT (right $p=0.013$, left $p=0.001$ ), PT/BW (right $p=0.008$, left $p=0.001$ ) and TW (right $p=0.036$, left $p=0.004$ ) at 60 degrees/s. The AP increased significantly after treatment in the flexor muscles in the right knee $(p=0.009)$ and the extensor muscles in the left knee $(p=0.001)$. There were no significant differences in pre- and post-treatment ROM and $\mathrm{H} / \mathrm{Q}$ at 60 degrees/s and 180 degrees/s. CMT decreased pain and may improve extensor muscle strength in patients with knee OA, but does not appear to improve ROM. No adverse effects of CMT were reported in this study [1]. No adverse events were observed during this study and this method could be used as an alternative therapy in painful conditions.

\subsubsection{Fibromyalgia syndrome (FMS)}

In a study, 40 female patients with FMS were randomized into two groups, Relaxing Yoga (RY) and Relaxing Yoga plus Touch (RYT), for eight weekly sessions of stretching, breathing, and relaxing yogic techniques. RYT patients were further submitted to manipulative techniques of Tui Na. Several assessment tools were used to measure outcome. Only 33 patients completed the study. Both RY and RYT groups showed improvement in the Fibromyalgia Impact Questionnaire (FIQ) and visual analog scale (VAS) scores, which decreased on all sessions. The RYT group showed lower VAS and verbal scores for pain on the eighth session, but this difference was not maintained on the follow-up. Conversely, RY, VAS and verbal scores were significantly lower just on the follow-up. Passive alternative therapies may be useful in the improvement of patients with pain like FMS [17].

\subsubsection{Infantile cough}

The TCM theorized that the internal disease could be treated by external therapy, i.e., Tuina massage. The Tuina technique, an external method, harmonizes Zang-fu organs through regulating qi movement. In this observational study, fifty-one children (females $=32$, males $=19$, age three months to nine years) with cough received 'Three Steps to Regulate Qi Method' Tuina, once a day for five days. The step 1 regulates qi of the spleen and stomach. The step 2 regulates of the liver and lung, and step 3 regulates the qi of upper Jiao. Further details of this three step technique of Tuina are described. The effects were observed after five treatments. The dropout rate was zero. Among the 51 infantile patients, 37 cases were cured, 14 cases were improved, and the total effective rate was $100 \%$. The Tuina with three steps to regulate Qi is an effective method for the treatment of infantile cough. Yan-chao and colleagues suggested that Tuina in infants needs to be conducted gently along with highly-focused, steady breath and intensive monitoring of patients' reaction in case of any damage to the skin [18].

\subsubsection{Systematic review (SR) and meta-} analysis (MA) of low back pain

Low back pain (LBP) is a public health problem and causes greater financial burden on public attributed to direct and indirect costs. LBP needs effective but low cost therapies such as CAM. This systematic review and meta-analysis evaluate the effectiveness of Tuina-focused integrative Chinese medical therapies (TICMT) on inpatients with LBP. Through electronic searches of English and Chinese databases, randomized controlled trials (RCTs) of TICMT for in-patients with LBP were identified. PEDro scale was used to assess the methodological quality of the included RCTs and the meta-analyses of TICMT for LBP on pain and functional status were conducted. 20 RCTs were included. The methodological quality of the included RCTs was poor. The meta-analyses' results showed that TICMT had statistically significant effects on pain and functional status, especially Tuina plus Chinese herbal medicine and Tuina plus acupuncture. But Tuina plus moxibustion or hot pack did not show significant improvements on pain. The long-term evidence of TICMT was far from sufficient. Based on the results of included studies suggest that TICMT might be effective CAM treatments for in-patients with LBP. However, the poor methodological quality of the included RCTs means that high-quality RCTs with long follow-up are warranted. No adverse effects were reported in this study [19]. 


\subsubsection{Cervical radiculopathy: Systematic review and meta-analysis}

Radiculopathy, presented by neck pain and caused by degenerative progression is commonly encountered condition [20]. This review evaluates the current evidence of Chinese massage therapy (Tui $\mathrm{Na}$ ) for cervical radiculopathy. Electronic searches of 7 databases using keywords Tui $\mathrm{Na}$ alone Or Tui $\mathrm{Na}$ combined with conventional treatment $\mathrm{Or}$ radiculopathy, a number of RCTs were retrieved. Only five studies involving 448 patients were included. The pooled analysis from the 3 trials indicated that Tui $\mathrm{Na}$ alone showed a significant lowering immediate effect on pain score with moderate heterogeneity compared to cervical traction. The meta-analysis from 2 trials revealed significant immediate effects of Tui Na plus cervical traction in improving pain score with no heterogeneity compared to cervical traction alone. No adverse effect was reported. Tui $\mathrm{Na}$ alone or Tui Na plus cervical traction may be helpful to cervical radiculopathy patients, but supportive evidence seems generally weak. Future clinical studies with low risk of bias and adequate follow-up design are recommended [21]. These studies, systematic reviews and meta-analysis [4,13-23] have included many randomized clinical trials and observational researches, and review of all the studies is beyond the scope of this minireview. Overall, the level of evidence concerning included RCTs, SRs and MA on Tuina massage used in aforesaid diseases varies from level I to II to III.

\subsubsection{Other indications}

Other indications for Tuina combined with or without other CAM treatments or conventional medications include infantile diarrhea, nausea and vomiting, chronic fatigue syndrome, cancer-related hiccup, headache, migraine, scapulohumeral periarthritis, anxiety disorder, depression, insomnia, irritable bowel syndrome, premenstrual syndrome, flatus and bowel movement problems (constipation including induced by antipsychotic medications is relieved and shortened by massage), breast, lung and other cancers, other GIT discomfort including cancer, ADHD symptoms, sciatica and muscle spasm, cervical radiculopathy, essential hypertension, sacroiliac joint injury, infant eczema, improve lactation, pain symptoms, infantile anorexia, birth brachial plexus injury, infantile muscular torticollis and disc protrusion/herniation [4,18,22,24-34]. Overall, Tuina massage in conjunction with other traditional Chinese therapies and conventional medicines is used in many physical and psychiatric conditions with good outcome; however, the quality component of these studies remains a concern of most researchers.

\subsection{Adverse Effects of Tui $\mathrm{Na}$}

Tuina is reported to have mild side effects and some complications. Tuina is commonly used in conjunction with acupuncture, Chinese herbs and other TCM modalities. Side effects of Tuina may include short lasting mild headache, soreness or mild pain, mild stomach upset, sedation, transient increased frequency of urine, the buildup and release of pressure throughout the body and bruising. There is a long list of adverse effects or complications of Tuina but they occur very rarely. Fever as a complication may occur when three steps Tuina massage is conducted in infants with cough [18]. Haematuria as a result of contusion to the kidney is a reported complication of Orthopedic Tuina in a patient presented with lumber disc herniation [35]. Other adverse or complications reported in several studies are broken skin, bleeding, fractures [36], hematoma, the swelling of the neck and arms, posterior interosseous nerve palsy, rupture of internal organs such as uterus and colon, pulmonary embolism, the embolization of the left kidney, transient destructive thyrotoxicosis, ulceration and infection resulted in amputation of leg, pseudo-aneurysm of popliteal artery, displacement of ureteral stent, deep vein embolism of left lower extremity, syncope, pain, tiredness and death (Table 1), [26-53]. The cause of death concerning a 26-year old, Singapore patient remains a mystery [53]. For avoiding complications, patients must consult a medical doctor or a certified Tuina practitioner for clarifying whether or not he/she is suitable to receive Tuina therapy. Overall, caution needs to be exercised while performing Tuina massage on individual having diseases especially affecting neck, spine, chest, abdomen and legs.

\subsection{Contraindications}

There are many reported contraindications of using Tui $\mathrm{Na}$ (WHO, 2010, see Table 2). These contraindications are limited but not an exhaustive list. 
Table 1. Complications of Tuina therapy

\begin{tabular}{|c|c|c|c|}
\hline Disease & Tuina massage (points) & Complication & Outcome $^{*}$ \\
\hline Cough [18] & Tuina massage & Fever & Recovered \\
\hline Infant eczema [26] & Tuina massage & Broken/bruises skin & Recovered \\
\hline $\begin{array}{l}\text { Patient on anticoagulation } \\
\text { [38] therapy and a normal } \\
\text { woman [39] }\end{array}$ & $\begin{array}{l}\text { Digital massage and Deep } \\
\text { body massage }\end{array}$ & $\begin{array}{l}\text { Hematoma } \\
\text { Hepatic hematoma }\end{array}$ & Recovered \\
\hline Lumbar disc herniation [33] & Orthopedic Tuina & $\begin{array}{l}\text { Hematoma due to } \\
\text { contusion of kidney }\end{array}$ & Recovered \\
\hline Neck pain [40] & $\begin{array}{l}\text { Infrared heat and massage } \\
\text { therapy (neck and arm) }\end{array}$ & $\begin{array}{l}\text { Swelling of neck and } \\
\text { arms (myopathy) }\end{array}$ & Recovered \\
\hline Tennis elbow [41] & Friction massage (elbow) & $\begin{array}{l}\text { Posterior interosseous } \\
\text { nerve palsy }\end{array}$ & Recovered \\
\hline $\begin{array}{l}\text { Abdominal Pain due to fruit } \\
\text { stones \& constipation (to } \\
\text { entrapped wind) }[47,48]\end{array}$ & Massage (abdomen) & $\begin{array}{l}\text { Rupture of internal } \\
\text { organs (uterus and } \\
\text { colon) }\end{array}$ & Recovered \\
\hline $\begin{array}{l}\text { Leg pain due to below knee } \\
\text { thrombus [49] }\end{array}$ & Massaging(calf muscle) & Pulmonary embolism & Recovered \\
\hline Aortofemoral bypass[50] & $\begin{array}{l}\text { Massage \&walking (on } \\
\text { back) }\end{array}$ & $\begin{array}{l}\text { Embolization of } \\
\text { kidney(left) }\end{array}$ & Recovered \\
\hline Hashimoto's disease [52] & Massage (neck) & $\begin{array}{l}\text { Transient destructive } \\
\text { thyrotoxicosis }\end{array}$ & Recovered \\
\hline T2DM + PM [50] & $\begin{array}{l}\text { Vacuum boot foot massage } \\
\text { with mechanical device } \\
\text { (leg) }\end{array}$ & $\begin{array}{l}\text { Ulceration and infection } \\
\text { of leg }\end{array}$ & Amputation \\
\hline Neck pain [42] & $\begin{array}{l}\text { Electrical roller massage } \\
\text { device (neck) }\end{array}$ & Strangulation of neck & Death \\
\hline Exostosis on femur [43] & $\begin{array}{l}\text { Traditional Chinese } \\
\text { massage (leg) }\end{array}$ & $\begin{array}{l}\text { Pseudoaneurysm of } \\
\text { popliteal artery }\end{array}$ & Recovered \\
\hline Ureteral stent in place [44] & $\begin{array}{l}\text { Deep body massage using } \\
\text { Rolfing technique } \\
\text { (abdomen, pelvis, and } \\
\text { lower back) }\end{array}$ & $\begin{array}{l}\text { Displacement of } \\
\text { ureteral stent }\end{array}$ & Recovered \\
\hline Lumbar disc herniation [45] & $\begin{array}{l}\text { Deep manipulation } \\
\text { (lumber) }\end{array}$ & $\begin{array}{l}\text { Deep vein embolism of } \\
\text { left lower extremity }\end{array}$ & Recovered \\
\hline Various diseases [46] & Massage $^{* \star}$ (back) & $\begin{array}{l}\text { Syncope, pain, and } \\
\text { tired\# }\end{array}$ & Recovered \\
\hline Seizure [53] & $\begin{array}{l}\text { Tuina massage (two hours } \\
\text { session) }\end{array}$ & Developed seizures & $\begin{array}{l}\text { Died (cause } \\
\text { mystery) }\end{array}$ \\
\hline
\end{tabular}

\subsection{Investigation}

Tuina practice involves several conventional diagnostic methods $[5,15]$, which are various forms of imaging techniques [54], laboratory tests and physical and systemic assessments for excluding various aforesaid contraindications and recording the intervention progress through mapping neuronal activity and recovery from Tuina complications. However, these investigations are limited but certainly do not reflect a full list of tests.

\subsection{Training}

According to World Health organization (WHO) report (2010), Tui $\mathrm{Na}$ is used around the world. However, Tui $\mathrm{Na}$ practice needs continuous training of TN practitioners (or Tui Na doctors or Tui $\mathrm{Na}$ therapists). For this purpose, WHO developed a comprehensive curriculum for training TN professionals and paramedical personnel. As a result, the practice of TN could be streamlined worldwide. The benchmark of curriculum and training suggested by WHO is 
Table. 2 Contraindications of Tuina massage

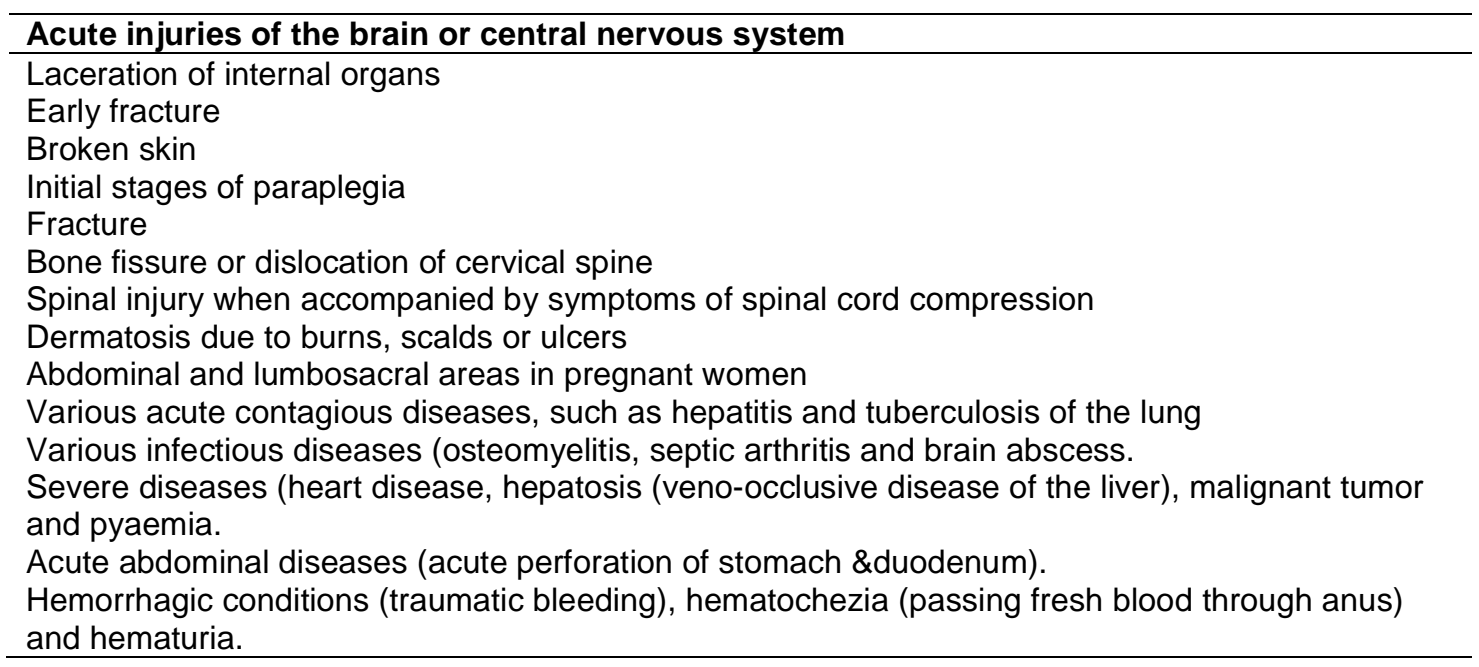

intended to equip trainees for professional treatment of commonly found painful symptoms or diseases of the musculoskeletal system. In addition, the prevention of complications of diseases associated with musculoskeletal, neurological and gynecological symptoms is also included in the curriculum. The program needs to be more organized to provide the trainees with important practical, professional, and theoretical information together with development of their management skills and competencies [5]. Curriculum should include principles of TCM, some components of western medicine, and other topics such as health regulations, ethical issues and research. As reported, many laypersons and unlicensed therapists practice Tui $\mathrm{Na}$ in many countries of the world resulting in poor outcomes of patients' conditions and poor quality of life [5]. Therefore, training in Tui Na for traditional practitioners across the world is crucial. Tui $\mathrm{Na}$ course needs to be integrated in academic universities, and traditional healthcare centers. Training is the first step to begin regulating Tuina practice. According to WHO philosophy, conversion programmes could be developed for practitioners with substantial basic health-care training to enable them to acquire the skills required to qualify for practicing Tuina. These programmes could be offered on either a full-time or a part-time basis, taking into accounts the trainee's background and previous health-care training. This comprehensive report can serve as a reference for national authorities wishing to establish systems of training, examination and licensure that support the qualified practice of Tuina [5]. Further details of training modules/benchmarks could be found in the subsequent section.

\subsection{Regulation}

The important components of underlying philosophy of regulating medical practice are ensuring safety of the patient, and quality of the service, continuous training of practitioners, examination and reevaluation of practitioners and licensing to those who fulfilled the criteria laid down by academic centers or medical/traditional governing bodies. The WHO [5] recognized benchmarks for training which suggested a list of topics for the training programs, methods of the training, and qualifications of the trainees and trainers. This WHO report also clarifies the roles and responsibilities of the future Tuina practitioners together with classification of Tuina programs according to the level of education of prospective trainees. This comprehensive document [5] suggested three types of program (type I, II and III programs). Type I training programs are directed at those who have completed high-school education or equivalent, but have no prior medical or other health-care training or experience. Type II training programs are targeted at those with medical or other healthcare training who intend to become recognized Tuina practitioners. Type III training program are aimed at practicing Tuina practitioners with either no prior medical or other health-care training, or inadequate training. Other details of three training programs are available here [5]. Further details of regulation, training and licensing concerning Tuina, see these sources $[55,56]$. 


\section{DISCUSSION}

Tuina is a Chinese heritage and has been used in Chinese culture since ancient time [1,2]. Tuina has a very rich history and is known by many terms which connote its literal meanings and possibly various defining principles, concepts and practices [3-5]. Over thousands of years, the scope of Tuina progressively increased not only in Chinese civilization but also in other cultures, and evidently this trend dynamically ongoing and enduring. At present, Tuina is used around the world and has many forms, techniques, procedures, and several underlying mechanisms of action [1,5,8-10]. In addition, Tuina is based on certain theories and principles [5,11-16].

Tuina is a well-recognized traditional Chinese massage therapy around the world and has innumerable clinical indications that are based on rigorous research evidence gathered through open and comparative randomized controlled clinical trials, systematic reviews and metaanalyses [1,5,8,13,17-22,24-34]. Furthermore, most of clinical research in which Tuina is used either alone or in conjunction with other CAM or conventional medicine reported positive results. Notably, the findings of the studies in which Tuina was used in combination with other therapies were more robust and significant compared to Tuina used standalone. However, most studies suggested that more rigorous researches with good quality criteria and design need to be conducted in future. Overall, Tuina has good clinical profile.

Tuina massage has many clinical applications and has been used safely in a variety of diseases with good outcome. Its clinical indications and contraindications are well recognized and reported in various studies [4,5,22-34]. Tuina is reported to have good safety profile, and most of the randomized controlled clinical trials along with systematic reviews and meta-analyses have reported no adverse effects [1,4,8,13-23]. However, some mild and transient side effects such as headache, nausea, vomiting, and pain have been reported in the literature. Notably, Tuina is associated with a variety of rare complications and deaths [5,18,26-53]. The diseases in which Tuina was used were heterogeneous when its complications occurred. Notably, only two deaths were reported in the literature [42,53], and the reason of death remained mysterious in one case [53]. She developed seizures during Tuina massage and died. Overall, despite good safety profile of Tuina massage, some mild adverse effects and major complications may occur rarely. Therefore, Tuina therapists need to exercise caution in excluding its contraindications, investigate the patients properly and only then Tuina massage is to be given to suitable patients.

Like conventional medicine providers, Tuina practitioners need continuous training. WHO team developed a comprehensive Tuina curriculum and also described guidelines concerning educational levels of prospective trainees [5]. In the same report, WHO experts also provided strategies for regulating Tuina practice around the world. In a nutshell, continuous training of Tuina practitioners and regulation of Tuina practice through registration and licensing impressively results in acquisition of core skills, good clinical practice and better outcome with less opportunity to adverse event occur.

This minireview has some limitations. As there is huge literature on Tuina in China and the rest of the world, selection and publication biases are the two caveats. Another related limitation is that screening of databases results may have excluded important articles on Tuina massage especially in Chinese language that have no English abstracts. The strength of this narrative review is that extensive electronic searches of databases were conducted in order to present the clinical characteristics of Tuina massage along with a comprehensive coverage on training and regulatory perspectives.

\section{CONCLUSION}

Evidently, Tuina is an ancient Chinese massage therapy, and is now effectively used either alone or combined with other therapies including complementary and alternative medicine or conventional medicine in a variety of diseases worldwide, and has minimal side effects and rare complications. Further studies on Tuina using rigorous quality assessment criteria and designs are warranted to provide strong and robust evidence-based data for its effectiveness in human diverse diseases.

\section{Notes adapted from (5)}

Tuina: In ancient times, Tuina was also called "An Mo", "An Qiao" or "Qiao Mo".

Yin: The theory of yin and yang was originated in China since ancient civilization. It mainly deals 
with the origins of the universe as well as the motion and variation of all things in the natural world.

Five elements: These are wood, fire, earth, metal and water. People in ancient China believed that these elements are indispensable to daily life and productive labor and that these five elements were keys to the normal variations in the natural world.

Qi: The classic Chinese philosophy signifies $q i$ as the primary state of the universe. The $Q i$, blood and body fluids, the essential substances for life activities, flow constantly inside the body and all originate from the body's internal organs/viscera.

Zang-fu: It is a collective term for internal organs which are divided into two major categories, namely the five zang-organs (i.e., the heart, liver, spleen, lungs and kidneys) and the six fu-organs (i.e., the gallbladder, stomach, small intestine, and large intestine, urinary bladder and sanjiao [the triple energizer]).

Meridians and channels: The meridians and collaterals are important components of the body. They are linear inform and subdivided into several levels of branches which are interconnected with each other and form into a network.

The eight guiding principles: These are yin and yang, internal and external aspects, cold and heat, and asthenia and zang-fu and help in the differentiation of syndromes.

\section{CONSENT}

It is not applicable.

\section{ETHICAL APPROVAL}

It is not applicable.

\section{COMPETING INTERESTS}

Authors have declared that no competing interests exist.

\section{REFERENCES}

1. Qingguang Z, Jianhua L, Min F, Li G, Wuquan S, Nan Z. Effect of Chinese massage (Tui $\mathrm{Na}$ ) on isokinetic muscle strength in-patients with knee osteoarthritis. Journal Traditional Chinese Medicine. 2016;36(3):314-320.

2. Eng ML, Lyons KE, Greene MS, Pahwa R. Open-label trial regarding the use of acupuncture and Yin TuiNa in Parkinson's disease outpatients: A pilot study on efficacy, tolerability, and quality of life. The Journal Alternative Complementary Medicine. 2006;12(4):395-399.

3. McCarthy M. Palpatory literacy, Chinese therapeutic bodywork (Tui $\mathrm{Na}$ ) and the remediation of head, neck and shoulder pain. Journal Bodywork and Movement Therapies. 2003;7(4):262-277.

4. Tao W-W, Jiang H, Tao X-M, Jiang $P$, Sha LY, Sun XC. Effects of Acupuncture, Tuina, Tai Chi, Qigong, and Traditional Chinese medicine five element music therapy on symptom management and quality of life for cancer patients: A meta-analysis. Journal Pain Symptom Management; 2016.

DOI: 10.1016/j.jpainsymman.015.11.027

5. World Health Organization: Benchmarks for training in traditional/complementary and alternative medicine-Benchmarks for Training in Tuina. WHO. Geneva 27. Switzerland; 2010.

6. Moher D, Liberati A, Tetzlaff J, Altman DG. The PRISMA group. Preferred reporting items for systematic reviews and metaAnalyses: The PRISMA statement. Plos Med. 2009;6(7):e1000097.

Available:http://journals.plos.org/plosmedic ine/article?id=10.1371/journal.pmed. 10000 97 DOI: 10.1371/journal.pmed1000097

7. Ding Q, Yan M, Zhou J, Yang L, Guo J, Wang $J$, et al. Clinical effects of innovative Tuina manipulations on treating cervical spondylosis of vertebral artery type and changes in cerebral blood flow. Journal Traditional Chinese Medicine. 2012;32(3): 1-2.

Available:http://dx.doi.org/10.1016/S02546272(13)60043-6

(Accessed on April 1, 2017)

8. Ilić D, Đurović A, Brdareski Z, Vukomanović A, Pejović V, Graji M. The position of the Chinese massage (Tuina) in clinical medicine. Vojnosanitetski Pregled. 2012;69(11):999-1004.

9. Fang L, Fang M. Research progress on the standardization of Chinese Tuina therapy: A short review. Chinese Journal Integrative Medicine. 2013;19(1):68-72.

DOI: 10.1007/s11655-011-0755-6. 
10. Qingguang Z, Fang M, Gong L, Shuyuna J, Wuquanc S, Jianhuac L, et al. Gait analysis of patients with knee osteoarthritis before and after Chinese massage treatment. Journal Traditional Chinese Medicine. 2015;35(4):411-416.

11. Souza DE, Franca, DLM, SennaFernandes V, Sá-Caputo DC, Guilhon $S$, Santos AAV, et al. On the Interconnectedness of Meridian System and Zang-Fu: A comparative review and its implications. Adaptive Medicine. 2014;6: 41-48.

12. Wang DJ, Lin CJ, Wang MZ, Xin MJ, Jing $Z$. Massage and rehabilitation. Liaoning Zhong Yi Za Zhi. 2009;36(2):189-190.

13. Aisong G, Qingyi $M$. Acupuncture combined with spinal Tui $\mathrm{Na}$ (or Tuina) for treatment of primary dysmenorrhea in 30 cases. Journal Traditional Chinese Medicine. 2008;28(1):7-9.

14. De-feng CAI. Warm-needling plus Tuina relaxing for the treatment of carpal tunnel syndrome. Journal Traditional Chinese Medicine. 2010;30(1):23-24.

15. Pang J, Cao YL, Guo CQ, et al. A retrospective analysis of clinical effect to Chinese Massage treatment on knee osteoarthritis. Shi Zhen Guo Yi Guo Yao 2013;24(8):1936-1937.

16. Liu CZ, Lei B. Effect of Tuina on oxygen free radicals metabolism in patients with chronic fatigue syndrome. Zhongguo Zhen Jiu. 2010;30(11):946-948.

17. da Silva GD, Lorenzi-Filho G, Lage LV. Effects of Yoga and the Addition of Tui $\mathrm{Na}$ in Patients with Fibromyalgia. The Journal Alternative Complementary Medicine. 2007;13(10):1107-1113.

18. Yan-chao W, Ting L, Dong-dong $Y$. Efficacy observation on Tuina for children with cough. Journal Acupuncture Tuina Science. 2015;13(6):387-390.

DOI: 10.1007/s11726-015-0888-9

19. Kong LJ, Fang M, Zhan HS, Yuan WA, Pu $\mathrm{JH}$, Cheng YW, Chen B. Tuina-focused integrative Chinese medical therapies for inpatients with low back pain: A systematic review and meta-analysis. Evidence-Based Complementary Alternative Medicine. 2012;14. Article ID 578305.

DOI: $10.1155 / 2012 / 578305$

20. Evans G. Identifying and treating the causes of neck pain. Medical Clinics North America. 2014;98(3):645-661.

21. Wei X, Wang S, Li L, Zhu L. Clinical evidence of Chinese massage therapy
(Tui $\mathrm{Na}$ ) for Cervical Radiculopathy: A systematic review and metaanalysis. Evidence-Based Complementary Alternative Medicine. 2017;2017:10. Article ID 9519285.

DOI: $10.1155 / 2017 / 9519285$

22. Ni X, Zhang-James Y, Han X, Lei S, Sun J, Zhou $R$. Traditional Chinese medicine in the treatment of ADHD: A review. Child adolescents psychiatric clinics North America. 2014;23:853-881.

Available:http://dx.doi.org/10.1016/i.chc.20 14.05. 011

(Accessed on February 27, 2017)

23. Cheng YH, Huang GC. Efficacy of massage therapy on pain and dysfunction in patients with neck pain: A systematic review and meta-analysis. Evidence Based Complementary Alternative Medicine. 2014;204360.

DOI: 10.1155/2014/204360

24. Yang $X$, Zhao $H$, Wang J. Chinese massage (Tuina) for the treatment of essential hypertension: A systematic review and meta-analysis. Complementary Therapy Medicine. 2014;22(3):541-548.

DOI: 10.1016/j.ctim.2014.03.008

25. Kuang JY, Li YX, He YF, Gan L, Wang AM, Tang SH, Lu FY, Yang LJ, Cat XL, Quan JL. Clinical therapeutic effect of oblique needling with Tuina in relieving sacroiliac joint injury. Zhen $\mathrm{Ci}$ Yan Jiu. 2016;41(2):169-174.

26. He YH, Kang J. Tuina massage for 120 cases of infants eczema. Zhongguo Zhen Jiu. 2012;32(12):1103-1104.

27. Lu P, Qiu J, Yao F, Zheng JJ. Effect of acupoint Tuina on lactation amount for parturient. Zhongguo Zhen Jiu. 2010; 30(9):731-733.

28. Tao WW, Jiang $H$, Tao $X M$, Jiang $P$, Sha LY, Sun XC. Effects of Acupuncture, Tuina, Tai Chi, Qigong, and Traditional Chinese medicine five-element music therapy on symptom management and quality of life for cancer patients: A meta-analysis. Journal Pain Symptom Management. 2016;51(4):728-47.

DOI: 10.1016/j.jpainsymman.2015.11.027

29. Wang YY, Liu XY. Clinical observation on fuzhong (supporting the middle-jiao) manipulation of tuina for infantile anorexia. Zhongguo Zhen Jiu. 2014;34(1):67-70.

30. Liu K, Wang Jie, Wang YG. Acupoint selection laws for massage therapy of 
infantile anorexia: An analysis based on data mining. Zhongguo Zhong Xi Yi Jie He Za Zhi. 2016;36(6):753-756.

31. Luo W, Cheng JZ. Clinical study on acupuncture and Tuina for treatment of birth brachial plexus Injury. Zhongguo Zhen Jiu. 2010;30(11):918-920.

32. Kang $\mathrm{Y}$, Lu S, Li J, Meng F, Chang $\mathrm{H}$. Primary massage using one-finger twining manipulation for treatment of infantile muscular torticollis. Journal Alternative Complementary Medicine. 2011;17(3):2317.

DOI: $10.1089 / \mathrm{acm} .2010 .0176$

33. Lei LM, Huang JJ, Lin GQ, Chen JS, Pang $\mathrm{YH}$, He YF, Tang HL, Gang W. Lumbar intervertebral disc protrusion treated with Santong tuina therapy: A multi-central randomized controlled trial. Zhongguo Zhen Jiu. 2011;31(3):253-257.

34. Every-Palmer S, Newton-Howes G, Clarke MJ. Pharmacological treatment for antipsychotic-related constipation. Cochrane Database Systematic Review. 2017;1:CD011128.

DOI.1465185/10.1002:CD 011128.pub2

35. Ying XM, Wang P, Yao BS, Gu HY, Xu QZ. Hematuria after orthopedic Tuina: A case report and brief review of literature. evidence-based complementary alternative medicine. 2011;2011:3. Article ID 953686. DOI: $10.1155 / 2011 / 953686$

36. Cheng WP. Basis of tuinaology, Chinese Publishing Company of Braille; 2007.

37. Ram SP, Kyaw K, Noor AR. Haematoma testes due to traditional massage in a neonate. Tropical Doctor. 1994;24(2):8182.

38. Yeo TC, Choo MHH, Tay MBE. Massive hematoma from digital massage in an anticoagulated patient: A case report. Singapore Medical Journal. 1994;35(3): 319-320.

39. Trotter JF. Hepatic hematoma after deep tissue massage. The New England Journal of Medicine. 1999;341(26):2019-2020.

40. Tanriover MD, Guven GS, Topeli A. An unusual complication: Prolonged myopathy due to an alternative medical therapy with heat and massage. Southern Medical Journal. 2009;102(9):966-968.

41. Wu YY, Hsu WC, Wang HC. Posterior interosseous nerve palsy as a complication of friction massage in tennis elbow.
American Journal Physical Medicine Rehabilitation. 2010;89(8):668-671.

42. Deidiker RD. Accidental ligature strangulation due to a roller-type massage device. American Journal Forensic Medicine Pathology. 1999;20(4):354-356.

43. Kalinga MJ, Lo NN, Tan SK. Popliteal artery pseudo-aneurysm caused by an osteochondral-a traditional medicine massage sequelae. Singapore Medical Journal. 1996;37(4):443-445.

44. Kerr HD. Ureteral stent displacement associated with deep massage. Wisconsin Medical Journal. 1997;96(12): 57-58.

45. Sheng JY, Li H, Huang MZ, Wu HJ. Deep vein embolism of left lower extremity due to deep manipulation Taxis treating lumbar disc herniation: A case report. Journal Zhejiang University Traditional Chinese Medicine. 1989;12(1):33.

46. Zhang SL, Hu QM. Protection of abnormal occurrence due to massaging by hung upside down in treatment of low back and leg pain. The Journal Traditional Chinese Orthopedics Traumatology. 2007;19(8):95.

47. Thambu JA. Rupture of the uterus: Treatment by suturing the tear. The Medical Journal Malaya. 1971;25(4):293294.

48. Rahman MN, McAll G, ChaiKG. Massagerelated perforation of the sigmoid colon in Kelantan. Medical Journal Malaysia. 1987; 42(1):56-57.

49. Warren SE. Pulmonary embolus originating below knee. The Lancet. 1978; 2(8083):272-273.

50. Mikhail A, Reidy JF, Taylor PR, Scoble JE. Renal artery embolization after back massage in a patient with aortic occlusion. Nephrology Dialysis Transplantation. 1997; 12(4):797-798.

51. Sørensen L, Ibsen KE. Purulent myofasciitis in a patient with diabetes treated with a vacuum boot by a zone therapist. Ugeskrift for Laeger. 1993; 155(27):2150-2152.

52. Tachi J, Amino N, Miyai K. Massage therapy on neck: A contributing factor for destructive thyrotoxicosis? Thyroidology. 1990;2(1):25-27.

53. Goy P. Woman, 26, dies during Tuina massage.

Available:http://www.straitstimes.com/sing apore/woman-26-dies-during-therapeuticmassage 
54. Zhang $\mathrm{H}$, Chen $\mathrm{H}$, Wang $\mathrm{H}$, Li D, Jia $\mathrm{B}$, Tan Z, Zheng B, Weng Z. Effect of Chinese tuina massage therapy on resting state brain functional network of patients with chronic neck pain. Journal Traditional Chinese Medical Sciences. 2015;2(1):6068.

Available:http://doi.org/10.1016/i.jtcms. 2015.10.001

(Accessed on February 20, 2017)

55. Licensing of massage therapists (Section 228). The $190^{\text {th }}$ General Court of the Commonwealth of Massachusetts; 2017.
Available:https://malegislature.gov/Laws/G eneralLaws/Partl/TitleXVI/Chapter112/Sect ion228

(Accessed on March 28, 2017)

56. Health Regulation Department. Dubai Health Authority. Traditional, complementary and alternative medicine; 2011.

Available:https://www.dha.gov.ae/Docume nts/HRD/Healthcare\%20 professionals Scope\%200f\%20Practice/SCOPE\%20OF \%20PRACTICE\%20FOR\%20TCAM\%20P RACTITONERS $\vee$ 01.pdf (Accessed on March 28, 2017)

(0) 2017 Al-Bedah et al.; This is an Open Access article distributed under the terms of the Creative Commons Attribution License (http://creativecommons.org/licenses/by/4.0), which permits unrestricted use, distribution, and reproduction in any medium, provided the original work is properly cited.

Peer-review history:

The peer review history for this paper can be accessed here: http://sciencedomain.org/review-history/19376 